\title{
Circumnuclear Star Formation in Spiral Galaxies from HST Images: NGC 4314
}

\author{
Cláudia Winge \\ Gemini Observatory, Southern Operations Center. c/o AURA, Inc. \\ Casilla 603, La Serena, Chile \\ Miriani G. Pastoriza \\ Depto. de Astronomia, IF/UFRGS. Av. Bento Gonçalves, 9500, CP \\ 15051, CEP 91501-970, Porto Alegre, RS, Brazil
}

\begin{abstract}
The first results of a study of the circumnuclear star-forming regions in HST broad band images of the nearby galaxy NGC4314 are presented. After modeling the bulge stellar population using ellipse fitting and subtracting the resulting model from the original images, aperture photometry of the individual star-forming regions in the ring was performed. For a total of 75 regions detected $3 \sigma$ above the background, we obtained mean colors $(\mathrm{f} 439-\mathrm{f} 569)=-0.72 \pm 0.27$, and $(\mathrm{f} 569-\mathrm{f} 814)=$ $-0.69 \pm 0.35$, with the NW regions being $\sim 0.5$ mag bluer in both colors than the NE ones, an effect that may be caused by internal reddening.
\end{abstract}

\section{Introduction}

NGC 4314 is a nearby (for $\mathrm{H}_{\circ}=75 \mathrm{~km} \mathrm{~s}^{-1} \mathrm{Mpc}^{-1}, \mathrm{D}=9.7-13.4 \mathrm{Mpc}$ depending on the distance assumed to the Coma I Cloud) SBa galaxy with a LINER nucleus and a broken ring of star forming regions with radius $\sim 8^{\prime \prime}$. We have used HST/WFPC2 archival images in the F439W, F569W and F814W filters to analyze both the bulge and ring stellar content. The images have been corrected for Galactic reddening assuming $\mathrm{E}(\mathrm{B}-\mathrm{V})=0.025$.

The left panel of Figure 1 shows the F439W sharp divided image. The star forming regions in the ring and the complex structure of dust lanes and clumps both outside and inside the ring are clearly visible. The (f439-f814) color image (right panel) reveals the presence of bluer spiral arms outside the ring, with (f439-f814) and (f569-f814) average colors of $\sim-0.50$ and $\sim-0.25$, respectively. Inside the ring, the average colors are $(\mathrm{f} 439-\mathrm{f} 814)=0.38 \pm 0.12$ and $(\mathrm{f} 569-\mathrm{f} 814)=0.06 \pm 0.07$.

\section{Surface brightness profiles}

Figure 2 shows the surface brightness and color index profiles along and across the major axis of the external bar $\left(\mathrm{PA}=149^{\circ}\right)$ taken from the original images. The outer blue arms can be seen in the major axis profile, while the one across the bar reveals the dust lanes outside the ring. Note the relative constant col- 

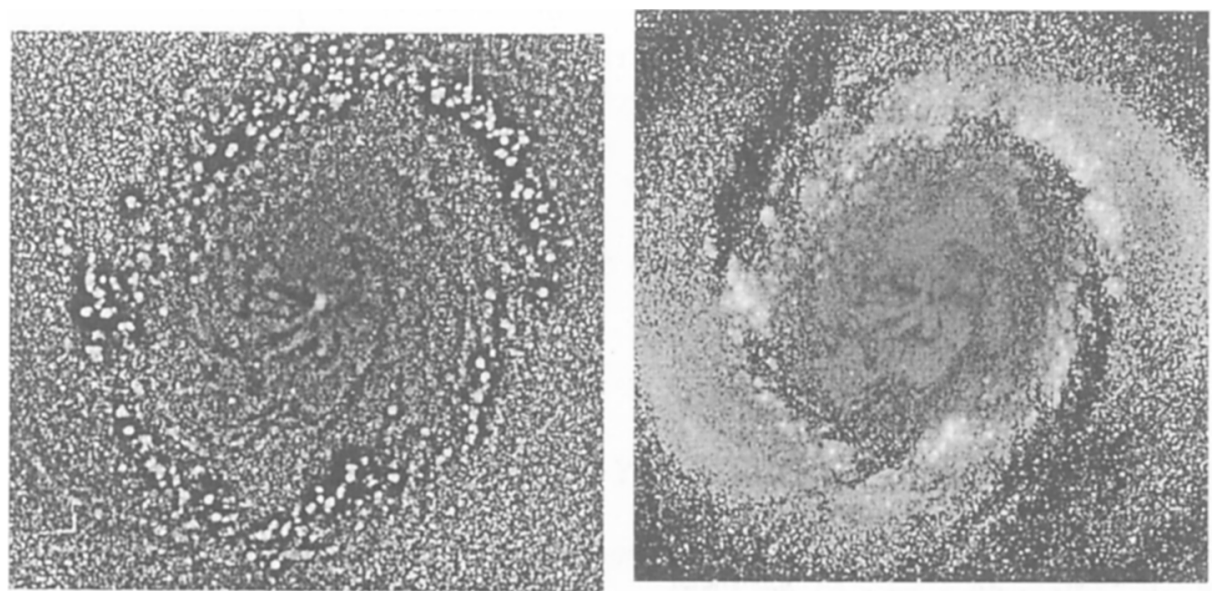

Figure 1. Left: F439W sharp-divided image. Note the dust spiral pattern all the way to the nucleus and the several pieces that compose the star-forming ring. North is up, East to the left, and the frame is $16^{\prime \prime}$ across. Right: (f439-f814) color image, showing the presence of the bluer spiral arms outside the ring. Orientation as the previous image, the field is $20^{\prime \prime}$ across.

ors inside the ring, disturbed by the clumpy distribution of dust. The profiles are also quite symmetric both along and across the bar, with the exception of F439W, which shows a marked asymmetry in the inner $2^{\prime \prime}$.

\section{The star forming ring}

The bulge emission up to $20^{\prime \prime}$ of the nucleus was modeled using ellipse fitting after masking both the emission and absorption features, and the model image subtracted from the original data. We then measured the luminosity of the individual emission regions in the ring using aperture photometry with the iterative guiapps.xguiphot IRAF task. The aperture radius was set for each region to include all the emission above a sky level set to $3 \sigma$ of the residual background in the outer parts of the frame, but avoiding contamination by nearby regions.

The results are shown in Figure 3, with the different colors identifying the main star forming complexes along the ring. The sample has mean F569W and F814W magnitudes of $14.9 \pm 0.9$ and $15.6 \pm 0.9$, and mean colors $(\mathrm{f} 439-\mathrm{f} 569)=$ $-0.72 \pm 0.27,(\mathrm{f} 569-\mathrm{f} 814)=-0.69 \pm 0.35$. There is a $\sim 0.5$ mag difference in both colors between the regions in the NW and NE strings, but without an estimate of the local reddening, we cannot say it this difference is due to obscuration or actual age variations. 

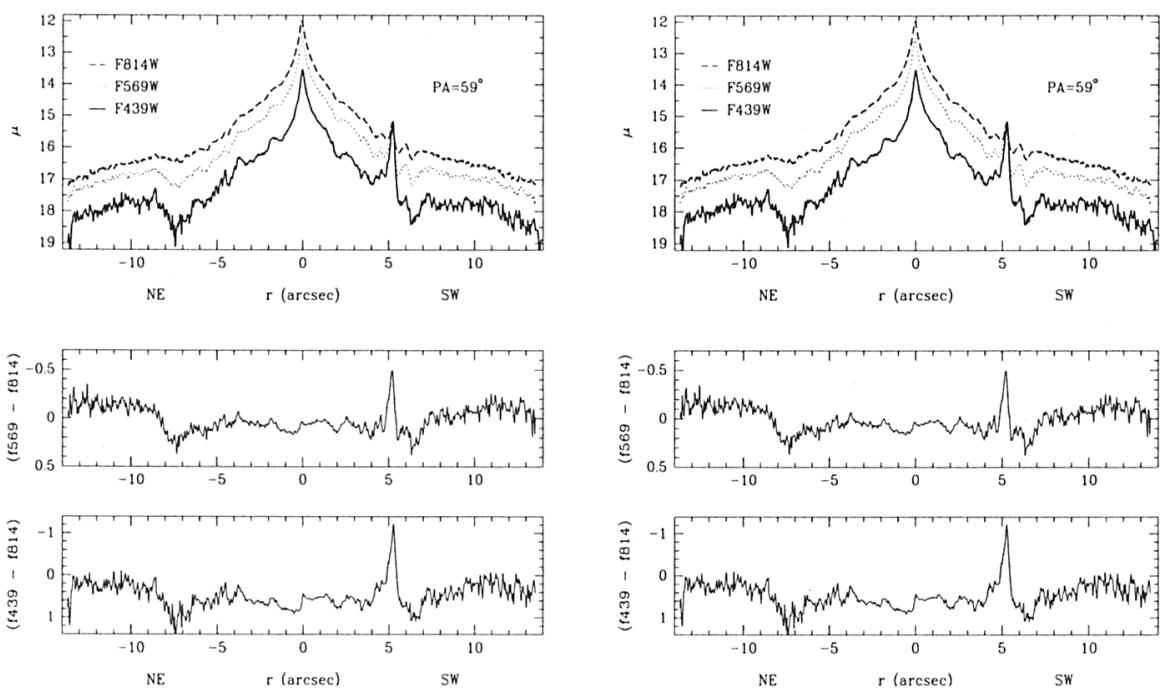

Figure 2. Surface brightness and color index profiles along the major (PA $=149^{\circ}$, left) and minor (right) axis of the external bar. In the top panel of both figures, the F569W and F $814 \mathrm{~W}$ have been shifted by +0.5 and $+1 \mathrm{mag}$, respectively, relative to F $469 \mathrm{~W}$.
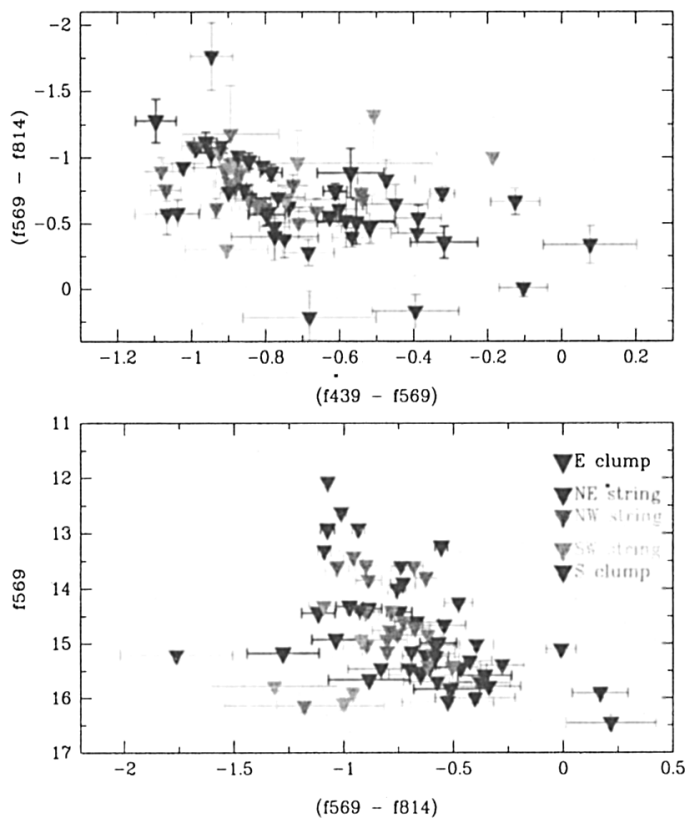

Figure 3. Color-color and color-magnitude diagrams for the starforming regions in the ring. These are observed colors and have not been corrected for internal reddening. 